\title{
INVERSE PROBLEMS IN ASTROPHYSICS
}

\author{
A. M. Cherepaschuk, A. V. Goncharski, \\ and A. G. Yagola \\ Physics Department, Moscow University \\ Sternberg State Astronomical Institute \\ USSR
}

\begin{abstract}
The applications of the regularizing algorithms to the solving of inverse problems in astrophysics are presented.
\end{abstract}

\section{INTRODUCTION}

The theory of incorrectly posed problems, created and advanced in the last years, has found applications for a wide range of inverse problems in optics, spectroscopy, electrodynamics, plasma diagnostics, geophysics, etc. (Tikhonov and Arsenin, 1977). Regularizing algorithms, when applied to the exerimental data processing, allow one to improve appreciably an accuracy of characteristics in studying physical phenomena. In some cases this is equivalent to increase of instrumental resolution. The increase of resolution is achieved because of computer employment in the data processing, rather than due to complication and development of the experimental installation itself, of ten connected with heavy expenses. The complex "device + computer" with the programming system of data processing, which involves regularising algorithms, has certainly great opportunities.

Astrophysics gives a large range of applications for solution of the "incorrectly posed problem" method, advanced in the last years. The matter is that presently astrophysics is basically an observational science. A researcher cannot exert an influence on processes taking place in distant stars and galaxies. He has to conclude about physical characteristics of such remote objects by their indirect manifestations visible from the Earth or near the Earth (from space stations). That is, an astrophysicist has to solve inverse problems while interpreting obtained observational data. Most of these problems are incorrectly posed. This means that a small change of experimental data can correspond with a however large change of a solution.

Since 1967 we have used stable methods (regularizing algorithms) advanced by the academician A. N. Tikhonov and his team (Tikhonov and Arsenin, 1977) for solving inverse (incorrectly posed) problems in astrophysics (Goncharsky, Cherepashchuk and Yagola, 1978). It needs to be said that investigation of specific astrophysical problems has stimulated development of some new mathematical methods. So, reconstructing the distribution of brightness over stellar disks in eclipsing binary systems, and using the natural physical information concerning monotonous decrease of the function to be determined, we have developed the effective numerical methods for solving incorrectly posed problems on sets of monotone functions (convex, monotone convex functions, and so on). The inverse problem for reconstruction of a strip-distribution of the radio-brightness over a source, which needs allowance for an error in representation of the radiotelescope directional pattern, has stimulated development of a theory and methods for solving incorrectly posed problems with an approximately specified operator. These methods have been realized as a complex of computer programs in FORTRAN (Tikhonov et al., 1983). Let us enumerate briefly astrophysical problems which have been solved by use of these and some other methods. The description of a problem statement and obtained results can be found in detail in our monograph Incorrect Problems in Astrophysics which will be published by "Nauka" press in 1985. 


\section{NATURE AND EVOLUTION OF WOLF-RAYET (WR) STARS IN CLOSE BINARY SYSTEMS}

We formulated the problem of the light curve interpretation in eclipsing binaries (including WR-components) from a new point of view. On the base of modern methods of incorrectly posed problems regularization the effective numerical algorithms have been developed for solving this problem on a computer. Moreover, the observations of all known binaries with WR-components have been carried out. In the result of light curve processing the geometrical parameters of the eclipsing systems with WR-components, as well as distributions of brightness and absorption properties over disc of WR stars have been determined. The nature and evolutionary stage of these objects have been studied.

\section{NEUTRON STARS AND BLACK HOLES IN BINARY SYSTEMS}

The special inverse problem algorithms for interpreting optical light curves of the X-ray binary systems have been developed in terms of a parametric model. These algorithms enable us to determine not only the values of parameters, but besides their confidence intervals. Xray binaries of various types were observed, including those in the southern sky. The analysis of these data by the new method yields the significant results. The lower limits for masses of relativistic objects in the systems Cygnus X-1 $\left(m_{x}>7 M_{0}\right)$, SS $433\left(m_{x}>6 M_{0}\right)$, LMC 3 $\left(m_{x}>7 M_{0}\right)$ are estimated. Thus, the number of candidates for black holes has increased from one to three during the last decade. This gives a real opportunity to state a problem of investigation of black holes on the observational basis. For most other X-ray systems (for example, HD 153919, HZ Her, HD 77581) the analogous computations yield mass estimates $\leq 2$ $M_{\odot}$ which is specific for a neutron stars. The optical parameters of accretion discs in X-rays binaries have been studied.

\section{CONCLUSION}

Moreover, the regularization method for solving incorrect problems has been used in following cases: a) for determining the observational structure and dynamical characteristics of accretion disks in nova and nova-like stars; b) for mapping a chemical element distributions over surface on Ap-stars (the problem was formulated by V. L. Khokhlova); c) for reconstruction of a strip-distribution of the brightness over a radio source.

Integration of the modern regularization methods for solving incorrect problems with observational practice in astronomy enables one to realize the effective regime of automated observations, when data processing and interpretations are carried out in real-time computations. An example of the problem which needs full automatization is the determination of a stellar angular diameters by the method of the lunar occultations of stars. At the High-Altitude expedition of SSAI (Sternberg State Astronomical Institute) the complex of equipment has been constructed which integrates a telescope with a computer and has a time resolution $10^{-3} \mathrm{~s}$.

In conclusion let us notice that applications of the regularization methods for solving incorrectly posed problems have led to a number of prospective directions in astrophysics.

\section{REFERENCES}

Goncharsky, A. V., Cherepashchuk, A. M., Yagola, A. G., The Numerical Methods for Solution of the Inverse Problems in Astrophysics. - Moscow, "Nauka", 1978.

Tikhonov, A. N., Arsenin, V. Ya., Solutions of III - Posed Problems, Scripta Series in Mathematics, Wiley, N. J., 1977.

Tikhonov, A. N., Goncharsky, A. V., Stepanov, V. V., Yagola, A. G., Regularizing Algorithms and a Priori Information. - Moscow, "Nauka", 1983. 